\title{
Long-term outcome of preadolescents, adolescents, and adult patients undergoing total cavopulmonary connection
}

Masamichi Ono, MD, PhD, ${ }^{\text {a }}$ Elisabeth Beran, MD, ${ }^{\mathrm{a}}$ Melchior Burri, MD, ${ }^{\mathrm{a}}$ Julie Cleuziou, MD, ${ }^{\mathrm{a}}$

Jelena Pabst von Ohain, MD, PhD, ${ }^{a}$ Martina Strbad, MS, ${ }^{a}$ Christoph Röhlig, MD, ${ }^{b}$ Alfred Hager, MD, PhD, ${ }^{b}$

Jürgen Hörer, $\mathrm{MD}, \mathrm{PhD},{ }^{\mathrm{c}}$ Christian Schreiber, $\mathrm{MD}, \mathrm{PhD},{ }^{\mathrm{a} \dagger}$ and Rüdiger Lange, $\mathrm{MD}, \mathrm{PhD}^{\mathrm{a}, \mathrm{d}}$

\section{ABSTRACT}

Objectives: Patients with a single ventricle infrequently undergo total cavopulmonary connection as preadolescents, adolescents, or adults. The purpose of this study was to clarify the characteristics of this cohort and to analyze the factors influencing outcomes.

Methods: Between 1994 and 2015, 50 of 460 patients underwent total cavopulmonary connection as preadolescents, adolescents, or adults (group A). The patients' characteristics and operative results were compared with those of the remaining 410 patients aged less than 9 years who underwent total cavopulmonary connection (group B). Post-total cavopulmonary connection echocardiogram reports $(\mathrm{n}=4862)$ were used to evaluate longitudinal ventricular function, and ejection fraction was characterized using nonlinear mixed-effects models and compared between groups.

Results: The median follow-up time was 10.3 (2.8-15.5) years. The differences between groups in 30-day mortality $(P=.20)$, intensive care unit stay $(P=.20)$, and incidence of prolonged effusion $(P=.08)$ were not significant. The estimated survival at 15 years was lower in group A $(86.5 \%)$ than in group B $(94.0 \%, P=.04)$ patients. Long-term systemic ventricular ejection fraction, analyzed with linear mixed-effect models, was significantly reduced in group A than in group $\mathrm{B}$ patients $(P<.001)$. At a median postoperative period of 8.4 (7.1-10.5) years, the peak oxygen uptake as measured by exercise capacity testing was lower in group A than in group B patients, respectively $(22.3 \pm 6.5[\mathrm{n}=25] \mathrm{vs}$ $30.6 \pm 8.1[\mathrm{n}=100] \mathrm{mL} / \mathrm{kg} / \mathrm{min}, P<.001)$.

Conclusions: The total cavopulmonary connection procedure was performed in preadolescent, adolescent, and adult patients with no significant difference in 30-day or hospital mortality compared with those in young children. However, long-term survival and ventricular performance were reduced in this older cohort. (J Thorac Cardiovasc Surg 2018;156:1166-76)

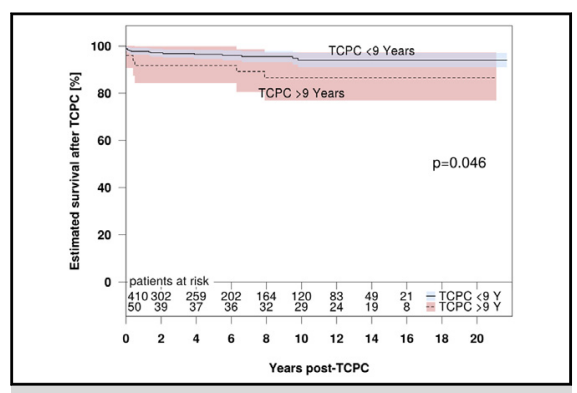

Freedom from death or transplantation was lower in the patients after delayed TCPC.

\section{Central Message}

TCPC was performed in preadolescent, adolescent, and adult patients with short-term results comparable to those for young children. However, long-term survival and late exercise capacity were reduced.

\section{Perspective}

Because of the late referral of patients, delayed TCPC was performed in preadolescent, adolescent, and adult patients. The short-term results were comparable to those of young children. However, long-term survival and late exercise capacity were reduced in these older patients, mainly because of impaired ventricular function and long-standing cyanosis. Therefore, an early TCPC is strongly recommended.

See Editorial Commentary page 1177.
From the Departments of ${ }^{\mathrm{a} C}$ Cardiovascular Surgery and ${ }^{\mathrm{b}}$ Pediatric Cardiology and Congenital Heart Disease, German Heart Center Munich at the Technical University of Munich, Munich, Germany; ${ }^{\mathrm{c}}$ Department of Congenital Heart Disease, Marie Lannelongue Hospital, Les Plessis-Robinson, France; and ${ }^{\mathrm{d}}$ German Centre for Cardiovascular Research, Munich, Germany.

${ }^{\dagger}$ Deceased.

This study was supported by grants from the Werner Reichenberger Foundation for Child Health.

Read at the 46th Annual Meeting of the German Society for Thoracic and Cardiovascular Surgery, Leipzig, Germany, February 11-14, 2017.

Received for publication May 15, 2017; revisions received March 15, 2018; accepted for publication March 22, 2018; available ahead of print May 10, 2018.

Address for reprints: Masamichi Ono, MD, PhD, Department of Cardiovascular Surgery, German Heart Center Munich at the Technical University, Lazarett Straße 36, Munich 80636, Germany (E-mail: ono@dhm.mhn.de).

$0022-5223 / \$ 36.00$

Copyright $($ c 2018 by The American Association for Thoracic Surgery

https://doi.org/10.1016/j.jtcvs.2018.03.155
In 1971, the "classic" Fontan operation consisting of an atriopulmonary connection was introduced for the surgical management of patients with a functional single ventricle. ${ }^{1}$ The original criterion for selecting patients included age more than 4 years but less than 16 years. $^{2}$ Approximately 20 years later, the "classic" Fontan operation was

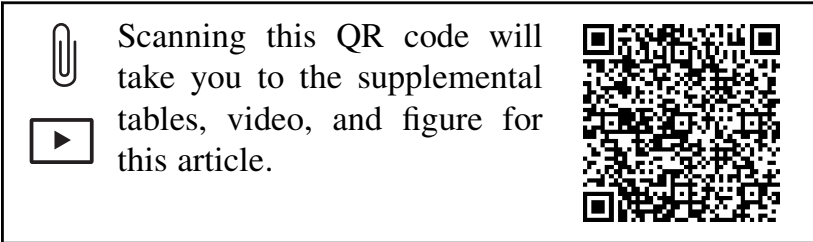



Abbreviations and Acronyms
$\mathrm{AVV}=$ atrioventricular valve
BCPS = bidirectional cavopulmonary shunt
$\mathrm{CoA}=$ coarctation of the aorta
df $=$ degrees of freedom
DILV = double inlet left ventricle
$\mathrm{EF} \quad=$ ejection fraction
ICU = intensive care unit
$\mathrm{PAB}=$ pulmonary artery banding
ROC = receiver operating characteristic
SVOT = systemic ventricular outflow tract
$\mathrm{TAPVC}=$ total anomalous pulmonary venous connection
TCPC = total cavopulmonary connection
$\mathrm{TV}=$ tricuspid valve
$\mathrm{VO}_{2}=$ oxygen uptake

sought to determine the clinical characteristics, perioperative outcomes, and long-term results for this cohort, along with the potential risk factors for ventricular function, mortality, and morbidities.

\section{PATIENTS AND METHODS}

The Institutional Review Board of the Technical University of Munich approved this study. The hospital charts, laboratory data, operative reports, catheterization results, and echocardiograms of these patients were carefully reviewed. Follow-up data were available for survivors and were obtained from the outpatient clinic at our institution or from transmissions from the satellite clinic, as well as by direct patient contact.

\section{Patients}

A total of 460 patients underwent TCPC at the German Heart Center Munich between May 1994 and December 2015. To clarify the features of the older patients, the 10th percentile of older patients (aged $\geq 9$ years) was selected $(n=50$, group A). The median age of group A patients at TCPC was 12.6 years (lower and upper quartiles: 10.0 and 15.8 years), and the oldest patient was 42 year old. Thirty-six patients $(72 \%)$ were referred from elsewhere. The patients' characteristics and outcomes were compared with those of the remaining 410 patients (aged $<9$ years at TCPC, group B).

\section{Operative Techniques and Postoperative Management}

The operative techniques for TCPC were described in our previous report. ${ }^{12}$ As for staged patients, the antegrade pulmonary blood flow was eliminated at the time of the BCPS for all patients. Fenestration was not routinely performed and was used only for very high-risk patients. ${ }^{12,23}$ Postoperatively, all patients were treated in the pediatric intensive care unit (ICU) regardless of their age. Since 2009, our institution has adopted a strategy of early extubation after TCPC. ${ }^{24}$

\section{Echocardiography}

The archived echocardiogram images from pre- and post-TCPC were reviewed. M-mode assessment of systemic ventricular function was performed, and the ejection fraction (EF) was calculated. ${ }^{25}$ Impaired ventricular function was defined if the EF was lower than $50 \%$. AVV regurgitation was graded as described in our previous study. ${ }^{9}$ Longitudinal echocardiographic data of various times after TCPC for each patient were collected, and the impact of age at TCPC on postoperative ventricular function was analyzed using linear mixed-effects models.

\section{Cardiopulmonary Exercise Capacity Testing}

A symptom-limited cardiopulmonary exercise test on a bicycle in an upright position was performed according to our institutional protocol, ${ }^{26}$ when the patients had reached 8 to 10 years of age. Peak oxygen uptake $\left(\mathrm{VO}_{2}\right)$ was defined as the highest mean uptake of any 30-second time interval during exercise. Age-, sex-, height-, and body mass-related reference values were calculated.

\section{Identification of Factors Affecting Ventricular Function, Mortality, and Morbidities}

To identify risk factors for ventricular dysfunction, mortality, and morbidities, we evaluated the variables related to the patient's primary diagnosis, cardiac morphology, type and number of palliative surgeries, and other preoperative conditions (Table E1).

\section{Statistical Analysis}

Continuous variables were expressed as means \pm standard deviations or as medians with interquartile ranges (Q1 to Q3), as appropriate. An lescent, adolescent, and adult patients (aged $\geq 9$ years). We 
independent sample $t$ test was used to compare normally distributed variables; the Mann-Whitney test was used for variables that were not normally distributed. The chi-square test was used for categoric data. Estimated survival and freedom from reoperation, intervention, and tachyarrhythmia were determined by the Kaplan-Meier method. Univariable risk analysis was performed by the log-rank test and ordinal logistic regression analysis. For multivariable analysis, we used the Cox proportionalhazards regression method to identify the variables that were independently predictive of events. Hazard ratios with $95 \%$ confidence intervals were estimated. Final models were derived by the forward and backward stepwise selection procedure. Variables with a level of significance of less than .1 in the univariable analysis were entered into the Cox and multiple logistic regression models. For statistical analysis of longitudinal ventricular function, linear mixed-effects models with a random intercept were used to account for repeated measures within patients. Natural cubic splines were used to model the potentially nonlinear relation of the continuous predictors "postoperative period" and "age at TCPC" to the outcome "EF." Therefore, the degrees of freedom (df) of the splines were varied from 1 , which corresponds to a linear effect, to 5 , which corresponds to a flexible nonlinear effect. Best subset selection guided by the Bayesian Information Criterion was used to select a model from all combinations of $\mathrm{df}$ for the splines as well as from models involving main effects only or additional interaction effects of the predictors. This selection resulted in a model of main effects only with $3 \mathrm{df}$ for the spline of "postoperative period" and $1 \mathrm{df}$ for the spline of "age at TCPC." Other variables were analyzed with the same methods. Hypothesis testing was performed on exploratory 2 -sided $5 \%$ significance levels. Receiver operating characteristic (ROC) curve analysis with the lower quartile of the $\mathrm{VO}_{2}$ was performed to determine the sensitivity and specificity of age for estimating exercise capacity. The Youden Index (J) was defined as follows: $\mathbf{J}=$ sensitivity + specificity -1 . The index was defined for all points on the ROC curve, and the maximum value of the index was used as a criterion for selecting the optimum cutoff point. The Statistical Package for the Social Sciences, version 22.0 for Windows (IBM, Ehningen, Germany) was used for analysis.

\section{RESULTS}

\section{Patients' Preoperative Characteristics and Features of Their Preoperative Conditions}

The preoperative characteristics of patients are shown in Table 1. Group A patients had more frequently received prior pulmonary artery banding (PAB) and less frequently BCPS surgery than group B patients. The morphology and function of the AVVs of all the study patients are summarized in Table 2. The prevalence of 2 morphologic AVVs and heterotaxy was higher in group A than in group B patients $(P=.021$ and $P<.001$, respectively) (Table E2). Systemic ventricular dysfunction $(\mathrm{EF}<50 \%)$ was observed more frequently in group A than in group B patients (10 patients $[20.0 \%]$ vs 29 patients [7.1\%], respectively; $P=.002)$. The parameters measured by preoperative cardiac catheterization are shown in Table 3.

\section{Type of Total Cavopulmonary Connection and Indication for Fenestration}

We performed a lateral tunnel TCPC in 50 patients from 1994 to 2001 (Table 4). During this period, 24 of the 50 patients $(48 \%)$ had undergone fenestration. Patients were considered as a "high-risk" candidate if more than 1 risk factor was present preoperatively. Risk factors included more than moderately impaired ventricular function, more than moderate AVV regurgitation, mean pulmonary arterial pressure greater than $15 \mathrm{~mm} \mathrm{Hg}$, mean atrial pressure greater than $12 \mathrm{~mm} \mathrm{Hg}$, pulmonary artery distortion, or any rhythm other than a sinus rhythm. In January 1999, we introduced the extracardiac TCPC at our institution. Gradually, construction of an intracardiac tunnel was abandoned. Since August 2001, we have performed only extracardiac TCPC, and the indication for fenestration was limited. Since 2002, only 5 patients underwent primary fenestration at the time of the TCPC (Table 4). Four of these patients had hypoplastic left heart syndrome associated with severe ipsilateral pulmonary artery atresia or hypoplasia. All patients underwent TCPC before the age of 9 years. The last patient had a heterotaxy syndrome with AVV regurgitation and a high mean pulmonary artery pressure after repair of the total anomalous pulmonary venous connection (TAPVC). This patient underwent TCPC at the age of 11 years. Since 2002 , in the 365 patients, the percentage of fenestration was $4.5 \%(1 / 21)$ in group $\mathrm{A}$ and $1.2 \%$ (4/339) in group B (Table 4).

\section{Operative and Postoperative Results of Group A Patients}

TCPC was performed with an extracardiac conduit in 41 patients and a lateral tunnel in 9 patients (Table 4). The mean cardiopulmonary bypass time was $124.6 \pm 53.4$ minutes. Twenty-five patients $(50.0 \%)$ required aortic crossclamping. The following concomitant procedures were performed: $10 \mathrm{AVV}$ procedures $(6$ tricuspid valve [TV] repairs, 2 TV closures, 1 TV replacement, 1 mitral valve repair), 10 pulmonary artery reconstructions, 8 pulmonary valve closures, 7 atrioseptectomies, and 1 Damus-Kaye-Stansel anastomosis.

The 30-day mortality of group A patients was $4.0 \%$ $(\mathrm{n}=2)$, which was not significantly different from that of group B patients $(1.5 \%[\mathrm{n}=6], P=.195)$. Among the group A patients, a 12-year-old girl presenting with double outlet right ventricle, mitral atresia, and coarctation of the aorta (CoA) died on postoperative day 16 after extracardiac TCPC. She had undergone 3 palliative operations before the TCPC procedure, namely, repair of the coarctation and $\mathrm{PAB}$, placement of a Blalock-Taussig shunt, and BCPS with patch enlargement of the left pulmonary artery at 3 months, 24 months, and 12 years of age, respectively. The postoperative course was complicated by marked low cardiac output, leading to thromboembolic closure of the hepatic vein and multiple organ failure. The other patient, a 16-year-old girl presenting with a single ventricle, mitral atresia, supracardiac TAPVC, and pulmonary stenosis, died on postoperative day 8 . She had undergone 4 previous palliative operations, namely, TAPVC repair, patch enlargement of the right pulmonary vein 
TABLE 1. Patient characteristics

\begin{tabular}{|c|c|c|c|}
\hline $\begin{array}{c}\text { Variables } \\
\text { N }(\%) \\
\end{array}$ & $\begin{array}{c}\text { TCPC } \geq 9 \text { y } \\
\text { (Group A) }\end{array}$ & $\begin{array}{c}\text { TCPC }<9 \text { y } \\
\text { (Group B) } \\
\end{array}$ & $P$ value \\
\hline No. of patients & $50(10.9)$ & $410(89.1)$ & \\
\hline Male sex & $28(56.0)$ & 143 (34.9) & .004 \\
\hline $\begin{array}{l}\text { Age at TCPC (y) } \\
\text { Median (IQR) } \\
\text { Minimum and maximum }\end{array}$ & $\begin{array}{c}12.6(10.0-15.8) \\
9.0-41.3\end{array}$ & $\begin{array}{c}2.2(1.7-3.0) \\
0.9-8.6\end{array}$ & $<.001$ \\
\hline $\begin{array}{l}\text { Weight at TCPC }(\mathrm{kg}) \\
\text { Median (IQR) } \\
\text { Minimum and maximum }\end{array}$ & $\begin{array}{c}38.1(29.7-48.7) \\
15.8-77.1\end{array}$ & $\begin{array}{c}11.5(10.5-13.4) \\
7.2-27.8\end{array}$ & $<.001$ \\
\hline $\begin{array}{l}\text { Primary diagnosis } \\
\text { UVH } \\
\text { DILV } \\
\text { ccTGA } \\
\text { PAIVS } \\
\text { TA } \\
\text { Unbalanced AVSD } \\
\text { HLHS } \\
\text { Others }\end{array}$ & $\begin{aligned} & 23(46.0) \\
& 12(24.0) \\
& 5(10.0) \\
& 3(6.0) \\
& 2(4.0) \\
& 0(0.0) \\
& 0(0.0) \\
& 5(10.0)\end{aligned}$ & $\begin{array}{c}98(23.9) \\
56(13.7) \\
17(4.1) \\
21(5.1) \\
69(16.8) \\
12(2.9) \\
110(26.8) \\
26(6.3)\end{array}$ & $\begin{array}{r}.001 \\
.052 \\
.067 \\
.792 \\
.018 \\
.220 \\
<.001 \\
.330\end{array}$ \\
\hline $\begin{array}{l}\text { Associated cardiac anomaly } \\
\text { TGA } \\
\text { DORV } \\
\text { CoA } \\
\text { Dextrocardia/situs inversus } \\
\text { Heterotaxy } \\
\text { TAPVC/PAPVC } \\
\text { Dominant right ventricle }\end{array}$ & $\begin{array}{r}30(60.0) \\
14(28.0) \\
6(12.0) \\
8(16.0) \\
10(20.0) \\
8(16.0) \\
27(54.0)\end{array}$ & $\begin{array}{r}155(37.8) \\
51(12.4) \\
57(13.9) \\
33(8.0) \\
27(6.6) \\
23(5.6) \\
212(51.7)\end{array}$ & $\begin{array}{l}.003 \\
.003 \\
.712 \\
.062 \\
.001 \\
.006 \\
.759\end{array}$ \\
\hline $\begin{array}{l}\text { Palliation and pre-Fontan condition } \\
\text { Aortopulmonary shunt } \\
\text { Blalock-Taussig shunt } \\
\text { PAB } \\
\text { Pacemaker implantation } \\
\text { Pulmonary artery reconstruction } \\
\text { AVV operation }\end{array}$ & $\begin{array}{c}15(30.0) \\
12(24.0) \\
19(38.0) \\
3(6.0) \\
4(8.0) \\
7(14.0)\end{array}$ & $\begin{aligned} 121 & (29.5) \\
123 & (30.0) \\
55 & (13.4) \\
9 & (2.2) \\
139 & (33.9) \\
39 & (9.5)\end{aligned}$ & $\begin{array}{r}.943 \\
.379 \\
<.001 \\
.111 \\
<.001 \\
.481\end{array}$ \\
\hline $\begin{array}{l}\text { No. of pre-TCPC surgeries } \\
0 \text { or } 1 \\
2 \text { or } 3 \\
4\end{array}$ & $\begin{array}{r}13(26.0) \\
30(60.0) \\
7(14.0)\end{array}$ & $\begin{array}{r}58(15.1) \\
315(82.0) \\
11(2.9)\end{array}$ & $<.001$ \\
\hline $\begin{array}{l}\text { Prior BCPS } \\
\text { Median age at BCPS (y and IQR) } \\
\text { Interval BCPS and TCPC (y and IQR) }\end{array}$ & $\begin{array}{l}31(62.0) \\
6.9(4.3-9.5) \\
5.9(2.1-8.2)\end{array}$ & $\begin{array}{l}380(92.7) \\
0.5(0.3-0.9) \\
1.5(1.2-2.1)\end{array}$ & $\begin{array}{l}<.001 \\
<.001 \\
<.001\end{array}$ \\
\hline
\end{tabular}

$T C P C$, Total cavopulmonary connection; $I Q R$, interquartile range; $U V H$, univentricular heart; $D I L V$, double inlet left ventricle; $c c T G A$, congenitally corrected transposition of the great arteries; PAIVS, pulmonary atresia and intact ventricular septum; TA, tricuspid atresia; $A V S D$, atrioventricular septal defect; $H L H S$, hypoplastic left heart syndrome; TGA, transposition of the great arteries; DORV, double outlet right ventricle; CoA, coarctation of the aorta; TAPVC, total anomalous pulmonary venous connection; PAPVC, partial anomalous pulmonary venous connection; $P A B$, pulmonary artery banding; $A V V$, atrioventricular valve; $B C P S$, bidirectional cavopulmonary shunt.

with resection of a subpulmonary stenosis, BlalockTaussig shunt placement, and redo-enlargement of the right pulmonary vein with placement of a central aortopulmonary shunt at the ages of 5, 6, 6, and 8 years, respectively. Extracardiac TCPC was performed, but massive TV regurgitation developed. The patient underwent TV replacement on postoperative day 8 , but died of ventricular dysfunction.
The remaining 48 patients survived the procedure through a 30 -day follow-up. Thirty-nine patients $(81.3 \%)$ were extubated within 24 hours postoperatively, and 30 patients $(62.5 \%)$ were discharged from the ICU within 7 days. The difference in the duration of ICU stay between the $2 \mathrm{pa}-$ tient groups was not significant (median 7.0 days in group A vs 8.0 days in group $\mathrm{B} ; P=.187$ ). Seven patients underwent redo thoracotomy for pericardial tamponade $^{5}$ and 
TABLE 2. Atrioventricular valve morphology and function

\begin{tabular}{lccc}
\hline $\begin{array}{c}\text { Variables } \\
\mathbf{N}(\%)\end{array}$ & $\begin{array}{c}\text { TCPC } \geq \mathbf{9} \\
(\text { Group A, } \mathbf{n}=\mathbf{5 0})\end{array}$ & $\begin{array}{c}\text { TCPC }<\mathbf{9} \\
(\mathbf{G r o u p} \mathbf{B}, \mathbf{n}=\mathbf{4 3 0})\end{array}$ & $\begin{array}{c}\boldsymbol{P} \\
\text { value }\end{array}$ \\
\hline AVV morphology & & & .015 \\
2 AVV & $24(48.0)$ & $129(31.5)$ & \\
MV & $7(14.0)$ & $101(24.6)$ & \\
TV & $11(22.0)$ & $144(35.1)$ & \\
CAVV & $8(16.0)$ & $36(8.8)$ & \\
Pre-TCPC AVVR & & & .023 \\
None & $13(26.0)$ & $158(38.5)$ & \\
Trivial & $23(46.0)$ & $130(31.7)$ & \\
Mild & $7(14.0)$ & $70(17.1)$ & \\
Moderate & $5(10.0)$ & $50(12.2)$ & \\
Severe & $2(4.0)$ & $2(0.5)$ & \\
\hline
\end{tabular}

$T C P C$, Total cavopulmonary connection; $A V V$, atrioventricular valve; $M V$, mitral valve; $T V$, tricuspid valve; $C A V V$, common atrioventricular valve; $A V V R$, atrioventricular valve regurgitation.

bleeding. ${ }^{2}$ Sixteen patients required late puncture of the thoracic cavity or reinsertion of chest tubes because of recurrent effusion. The difference in the rates of occurrence of prolonged pleural effusion ( $>7$ days) between groups was not significant $(30.0 \%$ in group A vs $41.8 \%$ in group $\mathrm{B}, P=.108$ ). Postoperative tachyarrhythmia did not develop in any patients. Temporary ventricular extrasystole developed in 1 patient, which had resolved by the time of discharge.

There were 16 patients with an interrupted inferior vena cava and azygos/hemi-azygos continuation, including 5 patients $(10 \%)$ in group A and 11 patients $(2.7 \%)$ in group B $(P=.008)$. Eleven of these patients had heterotaxy syndrome. Three patients in the early era underwent a 1-stage TCPC. The median age at TCPC was 3.3 (2.7-11.2) years. The median age for a Kawashima operation in 13 staged patients was $1.2(0.7-2.1)$ years, and the median interval between the Kawashima operation and TCPC was 2.2 (1.5-3.1) years. The size of the tube used for a hepatic vein inclusion was $14 \mathrm{~mm}$ in 1 patient, $16 \mathrm{~mm}$ in 4 patients, $18 \mathrm{~mm}$ in 8 patients, $20 \mathrm{~mm}$ in 2 patients, and $22 \mathrm{~mm}$ in 1 patient. No early death occurred within 30 days. One patient died of ventricular failure and multiorgan failure 4 months after the TCPC, and another patient died of prolonged hypoxia 6 years after the TCPC.

\section{Follow-up}

Of 48 early survivors, 3 patients $(6.3 \%)$ were lost to follow-up after hospital discharge. The median follow-up time was 12.5 (7.6-15.6) years. Late death occurred in 3 patients. The causes of death were ventricular failure, hypoxia due to intrapulmonary fistula, and sudden death during delivery (Table E3). One patient underwent heart transplantation 5 months after TCPC. The estimated overall 5 - and 15 -year survivals of group A patients were $91.7 \%$ and $86.5 \%$, respectively, which were significantly lower than the estimated survival of group B patients $(96.5 \%$ and $94.0 \%$, respectively; $P=.046$ ) (Figure 1). When age at TCPC was used as a continuous variable, age at TCPC was not identified as a significant risk factor for survival $(P=.060)$.

Late cardiac reoperations were required for 4 group A patients $(8.9 \%)$. The procedures included TV closure, fenestration closure, TCPC pathway revision, and pulmonary valve closure. The estimated freedom from cardiac reoperation at 15 years was $83.2 \%$, which was not significantly different compared with group B patients $(85.7 \%$, $P=.338)$. Late intervention was performed for 12 group A patients (26.7\%), including 7 collateral closures, 2 fenestration closures, 2 stent implantations, and 1 balloon angioplasty. The estimated freedom from intervention at 15 years was $61.6 \%$, which was not significantly different compared with group B patients $(72.4 \%, P=.589)$. Late-onset tachyarrhythmia was observed in 5 group A patients (11.1\%). The Kaplan-Meier estimated freedom from tachyarrhythmia at 15 years was $81.1 \%$ in group A patients, which was not significantly different compared with group B patients $(91.9 \%, P=.097)$.

The risk factors for mortality and morbidities in group A patients are shown in Table E4. The number of pre-TCPC

TABLE 3. Pre-total cavopulmonary connection catheter data and hemoglobin level

\begin{tabular}{|c|c|c|c|}
\hline Variables & $\begin{array}{c}\text { TCPC }>\text { y } \\
(\text { Group } \mathbf{A}, \mathbf{n}=\mathbf{5 0})\end{array}$ & $\begin{array}{c}\text { TCPC }<9 \text { y } \\
(\text { Group B, } n=430)\end{array}$ & $P$ value \\
\hline \multicolumn{4}{|l|}{ Pre-TCPC data } \\
\hline Hemoglobin $(\mathrm{g} / \mathrm{dL})$ & $18.0 \pm 2.8$ & $16.1 \pm 1.7$ & $<.001$ \\
\hline Mean pulmonary artery pressure $(\mathrm{mm} \mathrm{Hg})$ & $12.0 \pm 3.0$ & $9.2 \pm 3.0$ & $<.001$ \\
\hline Mean left atrial pressure $(\mathrm{mm} \mathrm{Hg})$ & $6.2 \pm 1.9$ & $5.4 \pm 2.4$ & .025 \\
\hline Transpulmonary gradient $(\mathrm{mm} \mathrm{Hg})$ & $5.1 \pm 2.6$ & $3.7 \pm 1.9$ & .001 \\
\hline Systolic ventricular pressure $(\mathrm{mm} \mathrm{Hg})$ & $91.1 \pm 15.6$ & $81.3 \pm 12.7$ & $<.001$ \\
\hline Ventricular endo-diastolic pressure $(\mathrm{mm} \mathrm{Hg})$ & $8.5 \pm 3.1$ & $7.3 \pm 2.8$ & .007 \\
\hline Aortic systolic pressure (mm Hg) & $90.0 \pm 15.8$ & $78.3 \pm 12.8$ & $<.001$ \\
\hline Aortic mean pressure $(\mathrm{mm} \mathrm{Hg})$ & $69.0 \pm 15.3$ & $55.4 \pm 9.2$ & $<.001$ \\
\hline Aortic oxygen saturation $(\%)$ & $84.2 \pm 5.4$ & $82.4 \pm 5.3$ & .029 \\
\hline Qp/Qs & $1.46 \pm 1.48$ & $0.79 \pm 0.49$ & .013 \\
\hline
\end{tabular}

$T C P C$, Total cavopulmonary connection; $Q p / Q s$, ratio of pulmonary-to-systemic flow. 
TABLE 4. Operative and postoperative outcomes

\begin{tabular}{|c|c|c|c|}
\hline $\begin{array}{c}\text { Variables } \\
\text { N }(\%) \\
\end{array}$ & $\begin{array}{c}\text { TCPC } \geq \mathbf{9} y \\
(\text { Group } A, \mathbf{n}=\mathbf{5 0})\end{array}$ & $\begin{array}{c}\text { TCPC }<9 y \\
(\text { Group B, } \mathbf{n}=\mathbf{4 3 0})\end{array}$ & $P$ value \\
\hline \multicolumn{4}{|l|}{ Operative data } \\
\hline \multicolumn{4}{|l|}{ Type of TCPC } \\
\hline Intracardiac (1994-2001) & $9(18.0)$ & $41(10.0)$ & .086 \\
\hline Extracardiac (1999-2015) & $41(92.0)$ & $369(90.0)$ & \\
\hline \multicolumn{4}{|l|}{ Conduit diameter $(\mathrm{mm})$} \\
\hline 14 & $0(0.0)$ & $1(0.3)$ & $<.001$ \\
\hline 16 & $1(2.4)$ & $6(1.6)$ & \\
\hline 18 & $5(12.2)$ & $336(91.1)$ & \\
\hline 20 & $21(51.2)$ & $25(6.8)$ & \\
\hline 22 & $14(34.1)$ & $1(0.3)$ & \\
\hline Median CPB time (min) & $115(84-101)$ & $61(44-95)$ & $<.001$ \\
\hline Aortic crossclamp & $25(50.0)$ & $111(27.1)$ & .001 \\
\hline Median aortic crossclamp time (min) & $69(40-94)$ & $42(26-68)$ & .003 \\
\hline Concomitant procedure & $25(50.0)$ & $128(31.2)$ & .008 \\
\hline Damus-Kaye-Stansel & $1(2.0)$ & $11(2.7)$ & .775 \\
\hline AVV procedure & $9(18.0)$ & $58(14.1)$ & .466 \\
\hline PA reconstruction & $11(22.0)$ & $43(10.5)$ & .017 \\
\hline Atrioseptectomy & $9(18.0)$ & $12(2.9)$ & $<.001$ \\
\hline SAS/VSD enlargement & $0(0)$ & $8(2.0)$ & .319 \\
\hline Pacemaker implant & $1(2.0)$ & $8(2.0)$ & .981 \\
\hline \multicolumn{4}{|l|}{ Fenestration at TCPC } \\
\hline Total & $9(18.0)$ & $26(6.3)$ & .003 \\
\hline Intracardial (1994-2001) & $7(77.8)$ & $17(41.5)$ & .048 \\
\hline Extracardiac (1999-2015) & $2(4.9)$ & $9(2.4)$ & .359 \\
\hline Period 1994-2001 & $8(28.6)$ & $22(32.8)$ & .684 \\
\hline Period 2002-2015 & $1(4.5)$ & $4(1.2)$ & .186 \\
\hline \multicolumn{4}{|l|}{ Postoperative data } \\
\hline 30-d mortality & $2(4.0)$ & $6(1.5)$ & .195 \\
\hline Hospital mortality & $3(6.0)$ & $9(2.2)$ & .111 \\
\hline \multicolumn{4}{|l|}{ Median intubation time (h) } \\
\hline Total & $14(9-27)$ & $8(4-16)$ & $<.001$ \\
\hline Period 1994-2008 & $19(10-48)$ & $15(10-24)$ & .214 \\
\hline Period 2009-2015 & $4(1-5)$ & $4(3-6)$ & .516 \\
\hline \multicolumn{4}{|l|}{ Median ICU stay (d) } \\
\hline Total & $8(5-11)$ & $7(5-9)$ & .227 \\
\hline Period 1994-2008 & $8(5-13)$ & $7(5-10)$ & .332 \\
\hline Period 2009-2015 & $7(5-7)$ & $6(4-8)$ & .690 \\
\hline Median hospital stay (d) & $25(19-30)$ & $20(14-27)$ & .012 \\
\hline Mean final CVP (mm Hg) & $13.9 \pm 1.8$ & $14.0 \pm 2.9$ & .082 \\
\hline \multicolumn{4}{|l|}{ Complications } \\
\hline Thorax revision & $7(14.0)$ & $14(3.4)$ & .001 \\
\hline Pleural effusion & $15(30.0)$ & $171(41.8)$ & .108 \\
\hline Chylothorax & $3(6.0)$ & $82(20.0)$ & .016 \\
\hline Ascites & $8(16.0)$ & $83(20.3)$ & .472 \\
\hline Follow-up echocardiography & $(\mathrm{n}=41)$ & $(\mathrm{n}=386)$ & \\
\hline Mild or moderate AVV regurgitation & $18(43.9)$ & $24(6.2)$ & .006 \\
\hline Reduced ventricular function & $11(26.8)$ & $24(6.2)$ & $<.001$ \\
\hline Cardiopulmonary exercise test & $(\mathrm{n}=25)$ & $(\mathrm{n}=100)$ & \\
\hline Postoperative period (y) & $8.5 \pm 4.2$ & $8.8 \pm 2.1$ & .713 \\
\hline Peak $\mathrm{VO}_{2}(\mathrm{~mL} / \mathrm{kg} / \mathrm{min})$ & $22.3 \pm 6.5$ & $30.6 \pm 8.1$ & $<.001$ \\
\hline Predicted peak $\mathrm{VO}_{2}(\%)^{*}$ & $63.8 \pm 10.4$ & $72.4 \pm 19.8$ & .048 \\
\hline
\end{tabular}

Variables are presented as $\mathrm{N}(\%)$ or median (interquartile range) or mean \pm standard deviation. $T C P C$, Total cavopulmonary connection; $C P B$, cardiopulmonary bypass; $A V V$, atrioventricular valve; $P A$, pulmonary artery; $S A S$, subaortic stenosis; $V S D$, ventricular septal defect; $I C U$, intensive care unit; $C V P$, central venous pressure; $V O_{2}$, oxygen uptake. *Percentage of age- and sex-related value. 


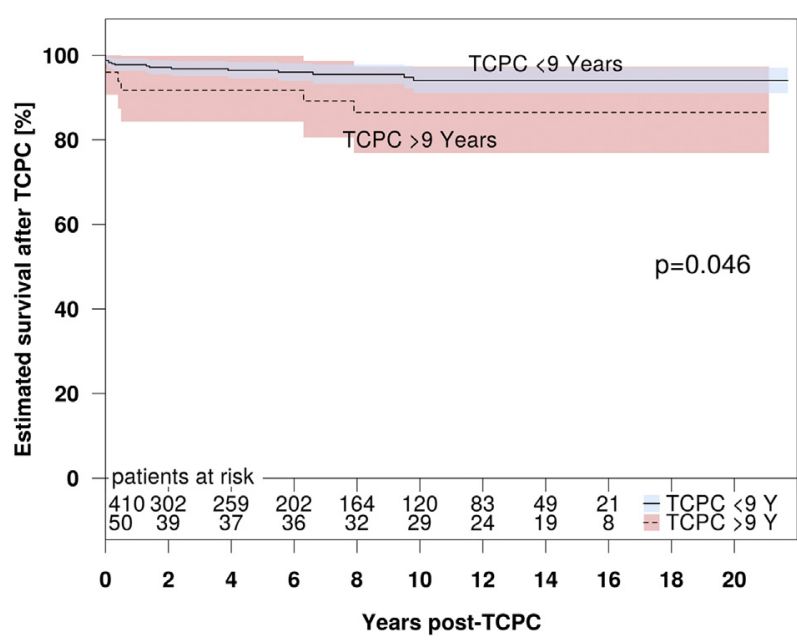

FIGURE 1. Kaplan-Meier estimate for freedom from death or transplantation in patients after TCPC: comparison of groups dependent on the age at TCPC. TCPC, Total cavopulmonary connection.

surgeries was identified as a risk for mortality $(P=.003)$. Dextrocardia was identified as a risk for reoperation $(P=.027)$, and heterotaxy was a risk for intervention $(P=.029)$. Risk factors for development of tachyarrhythmia could not be identified.

\section{Changes in Ventricular Function and Follow-up Echocardiography}

In group $\mathrm{A}$, of the 10 patients who presented with an impaired ventricular function preoperatively (EF: $43.3 \% \pm 3.3 \%)$, 4 recovered their function, but 6 continued to have impaired function (EF: $58.0 \% \pm 5.0 \%$ vs $38.7 \% \pm 4.3 \%$, respectively; $P<.001$ ). Of the 40 patients who presented with normal ventricular function preoperatively (EF: $64.0 \% \pm 8.2 \%$ ), 29 had preserved ventricular function and 11 had reduced ventricular function (EF: $61.9 \% \pm 7.3 \%$ vs $42.1 \% \pm 6.6 \%$, respectively; $P<.001$ ). Figure E1 demonstrates the changes in postoperative ventricular function for group $\mathrm{A}$.

To analyze the longitudinal ventricular function after TCPC in all patients, linear mixed-effects models were used. In total, 4862 post-TCPC echocardiogram reports were available during follow-up (mean 11 various times for each patient). Figure 2 demonstrates the longitudinal ventricular function. There was a significant difference in postoperative $\mathrm{EF}$ between group $\mathrm{A}$ and group $\mathrm{B}$ $(P<.001$, Figure 2, $A)$. When age at TCPC was used as a continuous variable, older age at TCPC was identified as a significant risk factor for postoperative lower EF $(P<.001$, Figure $2, B)$. The results of risk factor analysis for ventricular function are shown in Table 5. Age at TCPC was identified as a significant risk with univariate model $(P<.001)$, but it did not reach significance with the multivariate model $(P=.051)$. Pre-TCPC reduced ventricular function $(P<.001)$, pre-TCPC end-diastolic pressure $(P=.04)$, and PAB $(P=.04)$ were identified as significant risks with multivariable analysis.

Of 19 patients who had a prior PAB, 10 demonstrated post-TCPC ventricular dysfunction, and a systemic ventricular outflow tract (SVOT) obstruction was frequently observed in them (4 with CoA, 2 with subaortic stenosis, and 1 with restrictive bulboventricular foramen): Five patients demonstrated an SVOT gradient of $10 \mathrm{~mm} \mathrm{Hg}$ or more $(28,18,16,10$, and $10 \mathrm{~mm} \mathrm{Hg})$. However, as for the 9 patients with normal ventricular function after the TCPC, an SVOT lesion was observed in only 1 patient (CoA), and no patient demonstrated an SVOT gradient of more than $5 \mathrm{~mm} \mathrm{Hg}$.

Among 427 patients (41 group A and 386 group B patients) whose echocardiographic data on AVV regurgitation
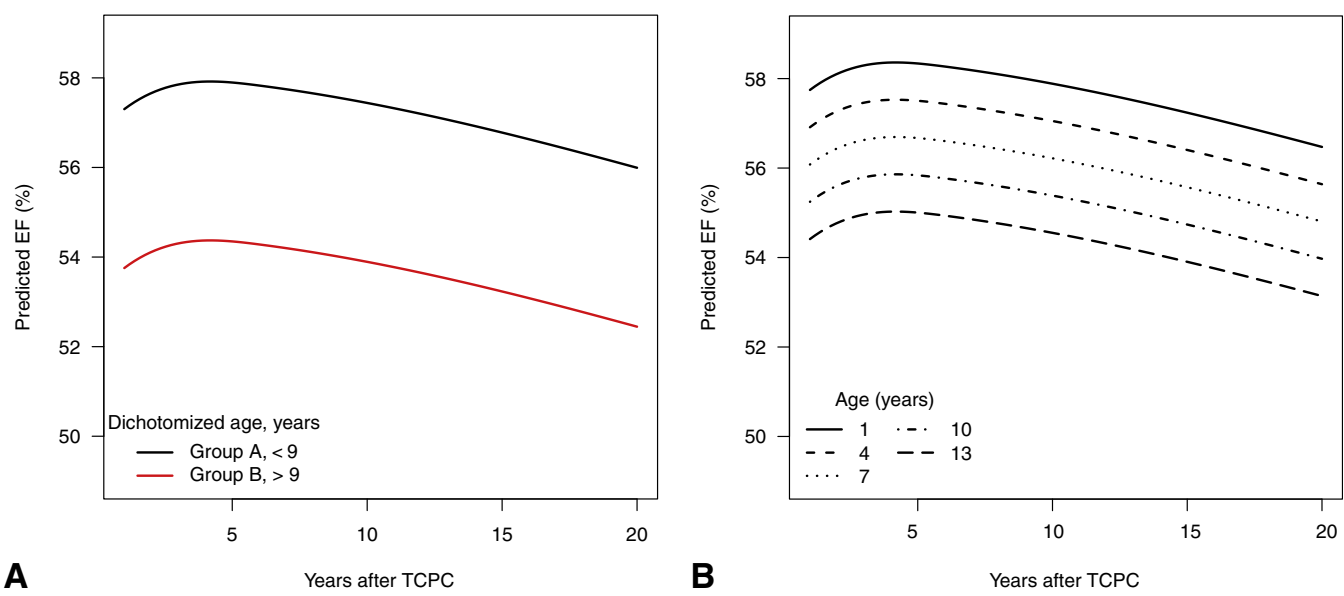

FIGURE 2. Longitudinal ventricular function after TCPC. A, Postoperative ventricular EF in group A was significantly lower than in group B $(P<.001)$. B, Older age at TCPC was a significant risk factor for postoperative lower $\mathrm{EF}(P<.001)$. TCPC, Total cavopulmonary connection; $E F$, ejection fraction. 
TABLE 5. Risk factors for longitudinal ventricular dysfunction with linear mixed-effect models

\begin{tabular}{|c|c|c|c|}
\hline \multirow[b]{2}{*}{ Variables } & \multirow{2}{*}{$\begin{array}{c}\text { Univariable } \\
\text { model }\end{array}$} & \multicolumn{2}{|c|}{$\begin{array}{l}\text { Multivariable } \\
\text { model }\end{array}$} \\
\hline & & $\overline{P \text { value }}$ & Coefficient \\
\hline Age at TCPC & $<.001$ & & \\
\hline $\begin{array}{l}\text { Pre-TCPC reduced } \\
\text { ventricular function }\end{array}$ & $<.001$ & $<.001$ & -8.459 \\
\hline AVV regurgitation & $<.001$ & & \\
\hline $\begin{array}{l}\text { Pre-TCPC end-diastolic } \\
\text { pressure }\end{array}$ & $<.001$ & .04 & 0.237 \\
\hline PAB & $<.001$ & .04 & -1.440 \\
\hline DILV & $<.001$ & & \\
\hline Single TV & .007 & & \\
\hline Prior AVV procedure & .014 & & \\
\hline $\begin{array}{l}\text { Transposition of the great } \\
\text { arteries }\end{array}$ & .026 & & \\
\hline No prior BCPS & .036 & & \\
\hline Dominant right ventricle & .045 & & \\
\hline Pre-TCPC hemoglobin level & .080 & & \\
\hline $\begin{array}{l}\text { Pre-TCPC mean pulmonary } \\
\text { artery pressure }\end{array}$ & .099 & & \\
\hline
\end{tabular}

were available at a median follow-up of 6.8 years (lower and upper quartiles: 2.6 and 12.0 years), mild or moderate AVV regurgitation was demonstrated in $18(43.9 \%)$ group A patients and $24(6.2 \%)$ group B patients $(P=.006)$.

\section{Follow-up Cardiopulmonary Exercise Testing}

A total of 125 patients underwent exercise testing on a bicycle in an upright position, according to our institutional protocol (25 group A and 100 group B patients). The measured peak oxygen uptake $\left(\mathrm{VO}_{2}\right)$ was significantly lower in group A than in group $\mathrm{B}$ patients $(22.3 \pm 6.5 \mathrm{~mL} / \mathrm{kg} / \mathrm{min}$ vs $30.6 \pm 8.1 \mathrm{~mL} / \mathrm{kg} / \mathrm{min}$, respectively; $P<.001$ ), as well as corrected peak $\mathrm{VO}_{2}$ values corrected for age and sex $(63.8 \% \pm 16.4 \%$ vs $72.4 \% \pm 19.8 \%$, respectively; $P=.048$ ) (Table 4 ). ROC analysis with lower quartiles (25th percentile) of the $\mathrm{VO}_{2}$ found that a cutoff age of 9 years had a specificity of 0.9 . The age of 2.8 years produced the maximum value for the Youden Index.

\section{DISCUSSION}

This study revealed that the TCPC procedure could be performed in preadolescent, adolescent, and adult patients, with short-term results comparable to those for young children. However, the long-term survival, ventricular function, and late exercise capacity of this older cohort were significantly worse (Video 1).

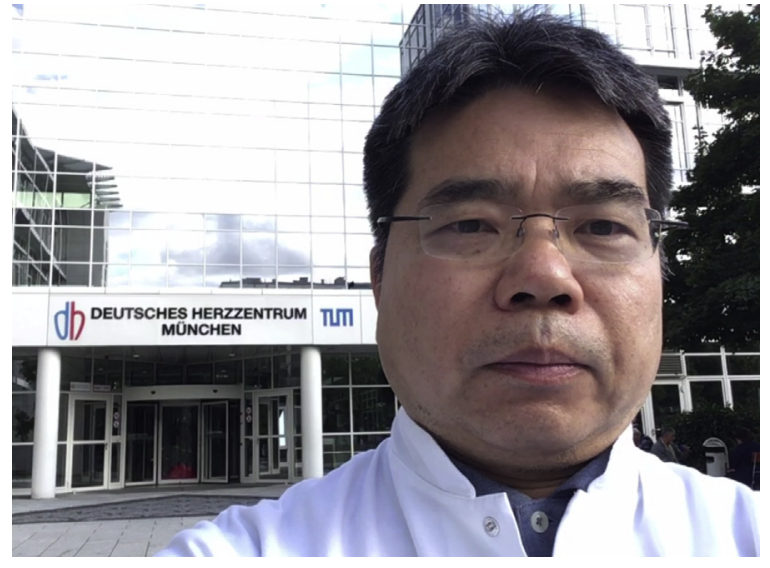

VIDEO 1. The author explains the importance and relevance of this study. Video available at: https://www.jtcvs.org/article/S0022-5223(18)30978-4/ fulltext.

\section{Cause of Late Referral, Indications for, and Short- Term Results of Late Total Cavopulmonary Connection}

In countries where advanced surgical procedures for congenital heart defects are available, the TCPC procedure is rarely performed in preadolescent, adolescent, and adult patients. In our series, the main cause of delayed TCPC was the late referral of patients from other countries or other states in Germany (accounting for $72 \%$ of referrals for group A patients).

Other than the completion of the Fontan circulation, few therapeutic options are available for older patients with a functional single ventricle. ${ }^{27}$ Heart transplantation carries a high early mortality and morbidity in patients with a single ventricle, and availability is limited because of the shortage of donor organs. We were able to show that completion of the Fontan circulation via the TCPC procedure is a viable option, even for older patients.

The indications for performing the TCPC procedure are essentially the same for both older and younger patients. Mean pulmonary artery pressure $(<20 \mathrm{~mm} \mathrm{Hg})$ and transpulmonary gradient $(<10 \mathrm{~mm} \mathrm{Hg})$ are the main decisive parameters for decision-making. Amount of left to right shunt volume is also taken into consideration for decisionmaking. At our institution, fenestration is not routinely performed and is limited to very high-risk patients, regardless of age. ${ }^{12}$ Stenosis of the pulmonary arteries and AVV regurgitation can be repaired before or at the time of TCPC.

The majority of group A patients appeared to tolerate the TCPC procedure very well during the first 30 days after surgery. The in-hospital mortality was similar to that of our younger patients. The incidence of unplanned reoperation was higher for older patients, mainly because of a bleeding complication. A longer cardiopulmonary bypass time and long-standing cyanosis (pre-TCPC: a higher hemoglobin level was observed in group A patients) might be the reason 
for this finding. Routinely, we leave in only 1 mediastinal drainage tube, but it might be better to leave in 2 or more drainage tubes in older patients. None of the patients developed postoperative atrial arrhythmia. Pace Napoleone and colleagues, ${ }^{28}$ who also reported the midterm findings after a modified Fontan procedure for 65 patients stratified by age into 2 groups, less than 7 years versus more than 7 years of age, showed results similar to ours. Fujii and colleagues ${ }^{18}$ reported 1 early death among 25 consecutive adult patients who had undergone a TCPC procedure, and Ly and colleagues ${ }^{16}$ reported that none of their 32 adult patients died in the hospital after TCPC.

\section{Long-Term Outcomes and Disadvantages of a Delayed Total Cavopulmonary Connection Procedure}

In this study, overall survival was significantly lower in patients who underwent a delayed TCPC procedure. Currently, the ideal surgical program for creating the Fontan circulation is a staged strategy of performing the BCPS procedure during early infancy followed by the TCPC procedure at 1 to 4 years of age. In general, all additional pulmonary blood flow is suppressed at the time of BCPS. $^{29}$ This strategy dramatically improves early and late survival to greater than $94 \%$ at 15 years postTCPC. ${ }^{10-12}$ In contrast, the survival of our older study cohort was $86.5 \%$ at 15 years. Talwar and colleagues ${ }^{30}$ demonstrated that the survival at 15 years was $84.7 \%$ in their 105 patients undergoing Fontan procedure at 10 years or older, very similar to our results. We assume that delayed completion of the Fontan circulation confers a significant disadvantage, mainly on systemic ventricular function. Our results showed that $20 \%$ of group A patients were found to have reduced preoperative ventricular function. Furthermore, longitudinal ventricular function analyzed by mixed-effects models revealed that older age at TCPC was a significant risk for worse postoperative ventricular function. In addition to these results, we found that late exercise capacity, which is a useful objective measure of ventricular functional ability, was significantly reduced in older patients. Because Qp/Qs at pre-TCPC catheterization was significantly higher in group A compared with group B and no prior BCPS was identified as a risk for postoperative ventricular dysfunction, long-standing volume loading of the systemic ventricle might cause impairment of ventricular function. Long duration of chronic cyanosis and repeated prior surgeries also cause ventricular dysfunction.

Of the 6 patients who died or received a heart transplantation, 4 required repeated surgeries for associated lesions (the TV, CoA, TAPVC, and right ventricular outflow tract). In a univariate model, previous AVV operation and CoA were also identified as risk factors for mortality. Therefore, the number of previous operations might serve as a surrogate for the risk of these associated lesions.

\section{Factors Affecting Systemic Ventricular Function}

In this study, risk factor analysis for ventricular dysfunction with mixed-effects models demonstrated that single TV was a stronger risk factor for ventricular function than dominant right ventricle ( $P=.007$ vs .045$)$. Our results suggest that the AVV morphology (presence of TV as a systemic $\mathrm{AVV}$ ), rather than the systemic ventricle morphology, is a more important factor influencing ventricular function. Uemura and colleagues ${ }^{31}$ showed that significant AVV regurgitation influenced systemic ventricular function after a Fontan operation. Anderson and colleagues ${ }^{32}$ demonstrated that semilunar and AVV regurgitation were more prevalent with right ventricular morphology and caused ventricular dysfunction. Wilder and colleagues ${ }^{33}$ reported that the right ventricular dysfunction begins to decline before BCPS, whereas tricuspid regurgitation does not peak until after BCPS and remained elevated at 6 years in their large multicenter study. Our previous study showed that AVV regurgitation was mild or less in $90 \%$ of patients with systemic left ventricle, whereas it was mild or less in $63 \%$ of those with systemic right ventricle and $58 \%$ of the patients with unbalanced atrioventricular septal defect or common inlet ventricles. $^{12}$ AVV regurgitation $(P<.001)$ and prior AVV procedure $(P=.014)$ were also identified as risks for ventricular dysfunction. In our study, 4 patients presenting with pre-TCPC ventricular dysfunction exhibited postoperative functional recovery. These 4 patients had no or mild AVV regurgitation. Our recent study demonstrated that impaired ventricular function recovers in half of the survivors after TCPC, and a competent AVV is a prerequisite for recovery. ${ }^{34}$ Other previous studies also demonstrated that AVV regurgitation is closely associated with ventricular function. ${ }^{10-12}$

PAB was also identified as a risk factor for post-TCPC ventricular dysfunction. Our results demonstrated a high association with SVOT obstruction for patients who had a prior $\mathrm{PAB}$ and a higher rate of ventricular dysfunction, compared with patients who had a PAB with preserved ventricular function. We assume that an SVOT obstruction is one of the main causes of systemic ventricular dysfunction after TCPC in this cohort. Alsoufi and colleagues ${ }^{35}$ demonstrated that $40 \%$ of patients with a PAB had a risk of an SVOT obstruction and recommended a Damus-Kaye-Stansel plus shunt or Norwood-type procedure as first-stage palliation. Our institutional policy has changed to include a Norwood-type procedure rather than a PAB for patients with double inlet left ventricle (DILV) and discordant ventriculoarterial connection, tricuspid type IIc, or mitral atresia and double outlet right ventricle.

\section{Optimal Timing of Completion for the Fontan Circulation}

The optimal timing for the TCPC procedure remains unclear. Investigators have reported that delayed Fontan 
surgery may be harmful, especially with regard to ventricular function and exercise capacity. ${ }^{7-9}$ We also demonstrated previously that exercise capacity was negatively correlated with age at TCPC and that younger age at TCPC was an independent predictor of better exercise capacity. ${ }^{9}$ The results of the present study support the assumption that delayed surgery may be harmful. Not only early unloading of the single ventricle but also early elimination of chronic cyanosis may be the most important factors involved in Fontan palliation for patients with a single ventricle. In this study, ROC analysis using cardiopulmonary exercise data demonstrated that the age of 2.8 years produced the maximum value of the Youden Index. We believe that early completion of the Fontan circulation might preserve systemic ventricular function, prevent (or at least mitigate) the harmful consequences of the Fontan circulation, and provide better functional ability.

\section{Study Limitations}

This study is limited by its retrospective, nonrandomized, single-center design. In addition, the small number of outcomes limits the statistical power of analysis. Data were not available at consistent follow-up periods for every patient. Qualitative assessment and grading are not the most accurate methods for evaluating ventricular function, and M-mode assessment of ventricular function in nonstandard-shaped ventricles is not a standardized method. Patients referred from other institutions might have a different history and quality of their remote medical and surgical management, and these factors could affect long-term outcomes. Changes over time in surgical and medical management might have affected the long-term outcomes. Changes in surgical management include the introduction of extracardiac TCPC since 1999 and nonfenestrated policy since $2001 .^{23}$ A tangible change regarding postoperative management was the adoption of an early extubation strategy even for unstable patients. ${ }^{24}$ Another limitation of the study was that not all patients had a recent cardiopulmonary exercise test. As for group B, many patients had not yet reached the age of 8 to 10 years, so a reliable cardiopulmonary exercise test could not be performed. Furthermore, some patients in both groups did not have regular follow-up in our congenital cardiologic outpatient department, so only medical reports from other institutions without exercise tests were available. The modest number of cardiopulmonary exercise tests might have limited the reliability of the results. The limited number of events might limit the reliability of the Cox regression results in some cases (end points).

\section{CONCLUSIONS}

The TCPC procedure performed in preadolescent, adolescent, and adult patients provided short-term results with no significant difference in 30-day or hospital mortality compared with those in young children. However, long-term survival and late ventricular performance might be reduced in these older patients, mainly because of impaired ventricular function.

\section{Conflict of Interest Statement}

Authors have nothing to disclose with regard to commercial support.

The authors thank Dr Alexander Hapfelmeier, Institute of Medical Information, Statistic and Epidemiology, Technical University of Munich, for assistance with the statistical analysis.

\section{References}

1. Fontan F, Baudet E. Surgical repair of tricuspid atresia. Thorax. 1971;26:240-8.

2. Choussat A, Fontan F, Besse P, Vallot F, Chave A, Bricaud H. Selection criteria for Fontan's procedure. In: Anderson RH, Shinebourne EA, eds. Pediatric Cardiology, 1977. Edinburgh: Churchill Livingstone; 1978:559-66.

3. de Leval MR, Kilner P, Gewillig M, Bull C. Total cavopulmonary connection: a logical alternative to atriopulmonary connection for complex Fontan operations. Experimental studies and early clinical experience. J Thorac Cardiovasc Surg. 1988;96:682-95.

4. Marcelletti C, Corno A, Giannico S, Marino B. Inferior vena cava-pulmonary artery extracardiac conduit. A new form of right heart bypass. J Thorac Cardiovasc Surg. 1990;100:228-32.

5. Kirklin JK, Blackstone EH, Kirklin JW, Pacifico AD, Bargeron LM Jr. The Fontan operation. Ventricular hypertrophy, age, and date of operation as risk factors. J Thorac Cardiovasc Surg. 1986;92:1049-64.

6. Gewillig M, Daenen W, Aubert A, Van der Hauwaert L. Abolishment of chronic volume overload. Implications for diastolic function of the systemic ventricle immediately after Fontan repair. Circulation. 1992;86(5 Suppl):II93-9.

7. Ovroutski S, Ewert P, Miera O, Alexi-Meskishvili V, Peters B, Hetzer R, et al. Long-term cardiopulmonary exercise capacity after modified Fontan operation. Eur J Cardiothorac Surg. 2010;37:204-9.

8. Madan P, Stout KK, Fitzpatrick AL. Age at Fontan procedure impacts exercise performance in adolescents: results from the Pediatric Heart Network Multicenter study. Am Heart J. 2013;166:365-72.

9. Ono M, Burri M, Cleuziou J, Pabst von Ohain J, Beran E, Strbad M, et al. Impact of early Fontan completion on postoperative outcomes in patients with a functional single ventricle. Eur J Cardiothorac Surg. 2017;51:995-1002.

10. Hirsch JC, Goldberg C, Bove EL, Salehian S, Lee T, Ohye RG, et al. Fontan operation in the current era: a 15-year single institution experience. Ann Surg. 2008; 248:402-10.

11. Nakano T, Kado H, Tatewaki H, Hinokiyama K, Oda S, Ushinohama H, et al. Results of extracardiac conduit total cavopulmonary connection in 500 patients. Eur J Cardiothorac Surg. 2015;48:825-32.

12. Ono M, Kasnar-Samprec J, Hager A, Cleuziou J, Burri M, Langenbach C, et al. Clinical outcome following total cavopulmonary connection: a 20-year single center experience. Eur J Cardiothorac Surg. 2016;50:632-41.

13. Grosse-Wortmann L, Drolet C, Dragulescu A, Kotani Y, Chaturvedi R, Lee KJ, et al Aortopulmonary collateral flow volume affects early postoperative outcome after Fontan completion: a multimodality study. J Thorac Cardiovasc Surg. 2012;144:1329-36.

14. Magee AG, McCrindle BW, Mawson J, Benson LN, Williams WG Freedom RM. Systemic venous collateral development after the bidirectional cavopulmonary anastomosis. Prevalence and predictors. J Am Coll Cardiol. 1998;32:502-8.

15. Duncan BW, Desai S. Pulmonary arteriovenous malformations after cavopulmonary anastomosis. Ann Thorac Surg. 2003;76:1759-66.

16. Ly M, Roubertie F, Kasdi R, Chatti S, Vergnat M, Luu D, et al. The modified Fontan procedure with use of extracardiac conduit in adults: analysis of 32 consecutive patients. Ann Thorac Surg. 2014;98:2181-6.

17. Roubertie F, Peltan J, Henaine R, Oses P, Iriart X, Thambo JB, et al. Early to midterm results of total cavopulmonary connection in adult patients. Ann Thorac Surg. 2013;95:941-7.

18. Fujii Y, Sano S, Kotani Y, Yoshizumi K, Kasahara S, Ishino K, et al. Midterm to long-term outcome of total cavopulmonary connection in high-risk adult candidates. Ann Thorac Surg. 2009;87:562-70. 
19. Mott AR, Feltes TF, McKenzie ED, Andropoulos DB, Bezold LI, Fenrich AL, et al. Improved early results with the Fontan operation in adults with functional single ventricle. Ann Thorac Surg. 2004;77:1334-40.

20. Ovroutski S, Alexi-Meskishvili V, Ewert P, Nürnberg JH, Hetzer R, Lange PE. Early and medium-term results after modified Fontan operation in adults. Eur J Cardiothorac Surg. 2003;23:311-6.

21. Veldtman GR, Nishimoto A, Siu S, Freeman M, Fredriksen PM, Gatzoulis MA, et al. The Fontan procedure in adults. Heart. 2001;86:330-5.

22. Gates RN, Laks H, Drinkwater DC Jr, Lam L, Blitz A, Child JS, et al. The Fontan procedure in adults. Ann Thorac Surg. 1997;63:1085-90.

23. Schreiber C, Hörer J, Vogt M, Cleuziou J, Prodan Z, Lange R. Nonfenestrated extracardiac total cavopulmonary connection in 132 consecutive patients. Ann Thorac Surg. 2007;84:894-9.

24. Georgiev S, Balling G, Ruf B, Ackermann K, Pabst von Ohain J, Schreiber C, et al. Early postoperative extubation of unstable patients following total cavopulmonary connection: impact on circulation and outcome. Cardiol Young. 2017;27: 860-9.

25. Margossian R, Schwartz ML, Prakash A, Wruck L, Colan SD, Atz AM, et al. Comparison of echocardiographic and cardiac magnetic resonance imaging measurements of functional single ventricular volumes, mass, and ejection fraction (from the Pediatric Heart Network Fontan Cross-Sectional Study). Am J Cardiol. 2009;104:419-28.

26. Müller J, Christov F, Schreiber C, Hess J, Hager A. Exercise capacity, quality of life, and daily activity in the long-term follow-up of patients with univentricular heart and total cavopulmonary connection. Eur Heart J. 2009;30:2915-20.

27. Deal BJ, Jacobs ML. Management of the failing Fontan circulation. Heart. 2012; 98:1098-104.

28. Pace Napoleone C, Oppido G, Angeli E, Giardini A, Resciniti E, Gargiulo G. Results of the modified Fontan procedure are not related to age at operation. Eur J Cardiothorac Surg. 2010;37:645-50.
29. Schreiber C, Cleuziou J, Cornelsen JK, Hörer J, Eicken A, Lange R. Bidirectional cavopulmonary connection without additional pulmonary blood flow as an ideal staging for functional univentricular hearts. Eur J Cardiothorac Surg. 2008;34: 550-4.

30. Talwar S, Singh S, Sreenivas V, Kapoor KS, Gupta SK, Ramakrishnan S, et al. Outcomes of patients undergoing primary Fontan operation beyond first decade of life. World J Pediatr Congenit Heart Surg. 2017;8:487-94.

31. Uemura H, Yagihara T, Kawashima Y, Yamamoto F, Nishigaki K, Matsuki O, et al. What factors affect ventricular performance after a Fontan-type operation? J Thorac Cardiovasc Surg. 1995;110:405-15.

32. Anderson PA, Sleeper LA, Mahony L, Colan SD, Atz AM, Breitbart RE, et al. Contemporary outcomes after the Fontan procedure: a Pediatric Heart Network multicenter study. J Am Coll Cardiol. 2008;52:85-98.

33. Wilder TJ, McCrindle BW, Phillips AB, Blackstone EH, Rajeswaran J, Williams WG, et al. Survival and right ventricular performance for matched children after stage-1 Norwood: Modified Blalock-Taussig shunt versus rightventricle-to-pulmonary-artery conduit. J Thorac Cardiovasc Surg. 2015;150: $1440-52$.

34. Ono M, Beran E, Burri M, Cleuziou J, Pabst von Ohain J, Röhlig C, et al. Outcomes of a total cavopulmonary connection in patients with impaired ventricular function. Eur J Cardiothorac Surg. January 22, 2018 [Epub ahead of print].

35. Alsoufi B, Manlhiot C, Ehrlich A, Oster M, Kogon B, Mahle WT, et al. Results of palliation with an initial pulmonary artery band in patients with single ventricle associated with unrestricted pulmonary blood flow. J Thorac Cardiovasc Surg. $2015 ; 149: 213-20$

Key Words: adolescents, adults, functional single ventricle, total cavopulmonary connection 


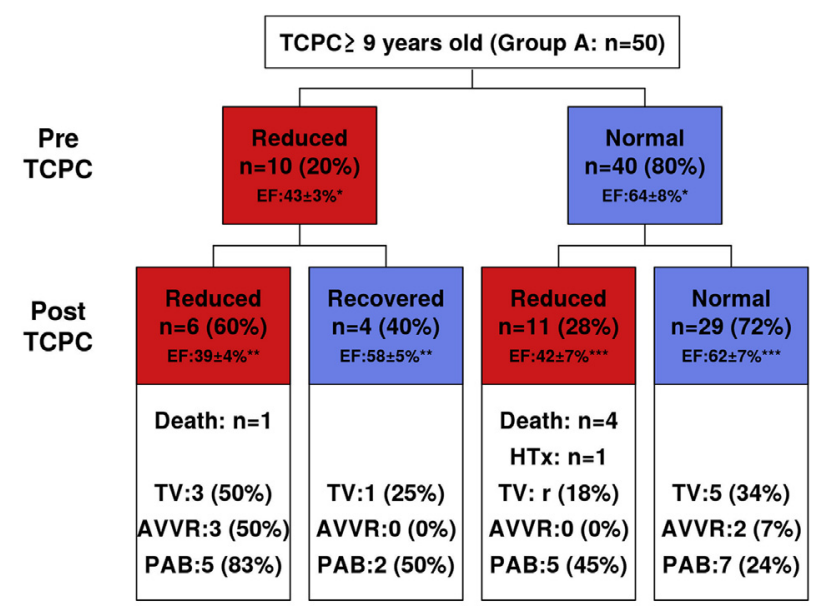

FIGURE E1. Tree diagram showing changes of ventricular function in group A patients (TCPC $\geq 9$ year old). $* P<.001$. $* * P<.001$. $* * * P<.001$. TCPC, Total cavopulmonary connection; $E F$, ejection fraction; $H T x$, heart transplantation; $T V$, tricuspid valve; $A V V R$, atrioventricular valve regurgitation; $P A B$, pulmonary artery banding. 
TABLE E1. Variables using for risk factor analysis

\begin{tabular}{l}
\hline \multicolumn{1}{c}{ Variables } \\
\hline Anatomic diagnosis \\
Congenitally corrected transposition of the great arteries \\
TA \\
DILV \\
PAIVS \\
AVV morphology \\
Two separate AVV \\
Mitral valve \\
TV \\
Common AVV
\end{tabular}

Associated anomaly

Transposition of the great arteries

Double outlet right ventricle

CoA

TAPVC

Dextrocardia

Heterotaxy syndrome

Dominant right ventricle

Palliative procedures

Aortopulmonary shunt

Blalock-Taussig shunt

PAB

Partial cavopulmonary connection

Pulmonary artery reconstruction

No. of pre-TCPC surgeries

Systemic ventricular function at TCPC

Reduced systemic ventricular function

History of reduced systemic ventricular function

AVV function at TCPC

AVV regurgitation

Prior AVV operation

Pulmonary vascular condition at TCPC

Mean pulmonary artery pressure $(\mathrm{mm} \mathrm{Hg})$

Mean left atrium pressure $(\mathrm{mm} \mathrm{Hg})$

Transpulmonary gradient $(\mathrm{mm} \mathrm{Hg})$

Single ventricle end-diastolic pressure $(\mathrm{mm} \mathrm{Hg})$

Prior pulmonary artery reconstruction

$T A$, Tricuspid atresia; $D I L V$, double inlet left ventricle; $P A I V S$, pulmonary atresia with intact ventricular septum; $A V V$, atrioventricular valve; $T V$, tricuspid valve; $C o A$, coarctation of the aorta; TAPVC, total anomalous pulmonary venous connection; $P A B$, pulmonary artery banding; TCPC, total cavopulmonary connection. 
TABLE E2. Morphologic factors presented in patients with total cavopulmonary connection aged more than 9 years

\begin{tabular}{|c|c|c|c|c|c|c|}
\hline \multirow[b]{2}{*}{ Variables } & \multicolumn{3}{|c|}{ Univariate model } & \multicolumn{3}{|c|}{ Multivariate model } \\
\hline & $\overline{P \text { value }}$ & OR & $95 \%$ CI & $P$ value & OR & $95 \% \mathrm{CI}$ \\
\hline DILV & .056 & 1.996 & $0.984-4.050$ & & & \\
\hline ccTGA & .076 & 2.569 & $0.905-7.294$ & & & \\
\hline $2 \mathrm{AVV}$ & .021 & 2.011 & $1.112-3.637$ & .021 & 2.367 & $1.276-4.391$ \\
\hline CAVV & .010 & 2.683 & $1.272-5.658$ & & & \\
\hline $\begin{array}{l}\text { Transposition of the great } \\
\text { arteries }\end{array}$ & .003 & 2.468 & $1.354-4.496$ & & & \\
\hline DORV & .004 & 2.737 & $1.382-5.422$ & & & \\
\hline TAPVC/PAPVC & .008 & 3.205 & $1.349-7.613$ & & & \\
\hline Dextrocardia & .068 & 2.176 & $0.944-5.018$ & & & \\
\hline Heterotaxy & .002 & 3.546 & $1.061-7.866$ & $<.001$ & 4.375 & $1.914-10.000$ \\
\hline
\end{tabular}

$O R$, Odds ratio; $C I$, confidence interval; $D I L V$, double inlet left ventricle; $c c T G A$, congenitally corrected transposition of the great arteries; $A V V$, atrioventricular valve; $C A V V$, common atrioventricular valve; DORV, double outlet right ventricle; TAPVC/PAPVC, total anomalous pulmonary venous connection/partial anomalous pulmonary venous connection.

TABLE E3. Mortality after total cavopulmonary connection

\begin{tabular}{|c|c|c|c|c|c|c|}
\hline Case & Diagnosis & $\frac{\text { Age at }}{\text { TCPC (y) }}$ & $\frac{\text { Type of }}{\text { TCPC }}$ & Concomitant procedure & Cause of death & Period \\
\hline \multicolumn{7}{|c|}{ 30-d mortality } \\
\hline 1 & DILV & 2 & $\mathrm{LT}$ & Damus-Kaye-Stansel & Cerebral infarction & $5 \mathrm{~d}$ \\
\hline 2 & AVSD & 1 & LT & AVV plasty & Cardiac failure & $2 d$ \\
\hline 3 & AVSD & 4 & $\mathrm{LT}$ & & Cardiac failure & $9 \mathrm{~d}$ \\
\hline 4 & SV, CoA & 12 & $\mathrm{EC}$ & & Hepatic vein thrombosis & $17 \mathrm{~d}$ \\
\hline 5 & SV, TAPVC & 16 & $\mathrm{EC}$ & & Cardiac failure, $\mathrm{MOF}$ & $8 \mathrm{~d}$ \\
\hline 6 & ccTGA, hypoplastic TV & 1 & $\mathrm{EC}$ & MV plasty, TV closure & Cardiac failure & $1 \mathrm{~d}$ \\
\hline 7 & DILV & 3 & $\mathrm{EC}$ & TV plasty, atrioseptectomy & Cerebral infarction & $7 \mathrm{~d}$ \\
\hline 8 & IAA, small LV & 2 & $\mathrm{EC}$ & & Cardiac failure & $5 \mathrm{~d}$ \\
\hline \multicolumn{7}{|c|}{ Late mortality } \\
\hline 1 & DILV, TGA & 5 & LT & PA plasty & PLE & $10 \mathrm{y}$ \\
\hline 2 & SV, TGA & 2 & LT & & Cardiac failure & $10 \mathrm{y}$ \\
\hline 3 & DIRV, DORV & 2 & $\mathrm{EC}$ & & PLE & $4 y$ \\
\hline 4 & SV, heterotaxy & 5 & $\mathrm{EC}$ & Damus-Kaye-Stansel & Cardiac failure, PLE & $1 \mathrm{y}$ \\
\hline 5 & SV, heterotaxy & 5 & $\mathrm{EC}$ & & Sepsis, MOF & $2 \mathrm{mo}$ \\
\hline 6 & PAIVS & 11 & $\mathrm{EC}$ & PA plasty, TV closure & Sudden death by delivery & $8 \mathrm{y}$ \\
\hline 7 & SV, heterotaxy & 18 & $\mathrm{EC}$ & AVV replacement & Cardiac failure, $\mathrm{MOF}$ & $4 \mathrm{mo}$ \\
\hline 8 & DILV & 3 & $\mathrm{EC}$ & & Cardiac failure & $7 y$ \\
\hline 9 & SV, heterotaxy & 10 & $\mathrm{EC}$ & & Hypoxia & $6 y$ \\
\hline 10 & HLHS & 1 & $\mathrm{EC}$ & TV replacement & Cardiac failure & $1 \mathrm{y}$ \\
\hline 11 & DORV, hypoplasty LV & 4 & $\mathrm{EC}$ & MV closure & Cardiac failure & $3 \mathrm{mo}$ \\
\hline 12 & TA Ic & 3 & $\mathrm{EC}$ & PV enlargement & Plastic bronchitis, MOF & $2 y$ \\
\hline 13 & TA Ic, CoA & 1 & $\mathrm{EC}$ & & Encephalopathy & $6 y$ \\
\hline 14 & $\mathrm{TA} \mathrm{Ib}$ & 1 & $\mathrm{EC}$ & & Viral infection & $2 \mathrm{mo}$ \\
\hline
\end{tabular}

$T C P C$, Total cavopulmonary connection; $D I L V$, double inlet left ventricle; $L T$, lateral tunnel; $A V S D$, atrioventricular septal defect; $A V V$, atrioventricular valve; $S V$, single ventricle; $C O A$, coarctation of the aorta; $E C$, extracardiac; $T A P V C$, total anomalous pulmonary venous connection; $M O F$, multiorgan failure; $c c T G A$, congenitally corrected transposition of the greater arteries; $T V$, tricuspid valve; $M V$, mitral valve; $I A A$, interrupted aortic arch; $L V$, left ventricle; $T G A$, transposition of the great arteries; $P A$, pulmonary artery; $P L E$, protein losing enteropathy; DIRV, double inlet right ventricle; DORV, double outlet right ventricle; PAIVS, pulmonary atresia intact ventricular septum; HLHS, hypoplastic left heart syndrome; $T A$, tricuspid atresia; $I c$, normally related great arteries and no infundibular or pulmonary stenosis. 
TABLE E4. Risk factors for mortality and morbidities in group A patients

\begin{tabular}{|c|c|c|c|c|c|c|}
\hline \multirow[b]{2}{*}{ Variables } & \multicolumn{3}{|c|}{ Univariate model } & \multicolumn{3}{|c|}{ Multivariate model } \\
\hline & $P$ value & HR & $95 \%$ CI & $P$ value & HR & $95 \%$ CI \\
\hline \multicolumn{7}{|l|}{ Mortality } \\
\hline Coarctation of the aorta & .097 & 4.217 & $0.769-23.126$ & & & \\
\hline Single TV & .082 & 4.148 & $0.834-20.617$ & & & \\
\hline $\begin{array}{l}\text { AVV operation before or at } \\
\text { TCPC }\end{array}$ & .069 & 4.837 & $0.885-26.430$ & & & \\
\hline No. of pre-TCPC surgeries & .003 & 2.119 & $1.284-3.498$ & .003 & 2.119 & $1.284-3.498$ \\
\hline \multicolumn{7}{|l|}{ Reoperation } \\
\hline Dextrocardia & .030 & 5.350 & $1.174-24.374$ & .027 & 6.136 & $1.223-30.775$ \\
\hline Transpulmonary gradient & .037 & 1.344 & $1.019-1.774$ & & & \\
\hline \multicolumn{7}{|l|}{ Intervention } \\
\hline Heterotaxy syndrome & .029 & 4.129 & $1.157-14.731$ & .029 & 4.129 & $1.157-14.731$ \\
\hline Blalock-Taussig shunt & .044 & 3.324 & $1.031-10.723$ & & & \\
\hline
\end{tabular}

$H R$, Hazard ratio; $C I$, confidence interval; $T V$, tricuspid valve; $A V V$, atrioventricular valve; $T C P C$, total cavopulmonary connection. 